\title{
Filtered Backstepping Control Based on the Nonlinear Disturbance Observer for Nonlinear System
}

\author{
Hui Wei ${ }^{1^{*}}$ \\ ${ }^{1}$ Department of Electronic Communication Engineering, Henan Institute of \\ Technology, Xinxiang, Henan 453003 China \\ wwggjd@126.com
}

\begin{abstract}
A filtered backstepping control method based on the nonlinear disturbance observer for a general class of multiple input multiple output high order nonlinear system with uncertainties is proposed. The uncertainty of the system can be estimated by nonlinear disturbance observer online. The filtered backstepping controller is designed based on the Lyapunov stability theorem which could avoid the the complex iterative derivative operation of supposition of traditional backstepping approach. A disturbance attenuation robust controller is adopted to decrease the influence of the control accuracy from the observer estimation error. Aiming at one kind of three-order nonlinear system with uncertainties, the simulation results illustrate that the nonlinear disturbance observer could estimate the composite disturbances of the system effectively and the disturbance attenuation method could improve the control accuracy while the observer existing error.
\end{abstract}

Keywords: Uncertain nonlinear system; filtered backstepping approach; nonlinear disturbance observer

\section{Introduction}

In recent years, the control problems for nonlinear systems with uncertainties have attract much attentions. To solve the control problem for nonlinear system with unknown disturbances, the fuzzy neural network based sliding mode control method is proposed in [1-2], the fuzzy neural network is used to estimate the unknown disturbances. In [3], the sliding mode control is used to solve the uncertainties. In [4], the $H_{\infty}$ control is used to deal with the disturbances.

In the above literatures, fuzzy system or neural networks are used to estimate the uncertainties of the system. Theoretically, neural network can approximate the nonlinear function with arbitrary accuracy, however, the problems such as slow convergence speed and large approximation error at initial time also exist. For the sliding mode control, the chattering problem makes the actuators very hard to response the control output. For the $H_{\infty}$ control theory, the conservatism makes it different to be used in practice.

Backstepping technique is an effective way to deal with the high order nonlinear system. However, the tradition backstepping approach need to calculate the derivative of the virtual controller, which makes this approach hard to be used in practice for high order system [10]. Disturbance observer (DOB) is an effect way to deal with the system uncertainties, it can estimate the system uncertainties online and thus compensate the system into the nominal one [5-8].

Based on the analysis above, the nonlinear DOB (NDOB) based command backstepping methodology is proposed in this paper. We first design the NDOB to estimate the system uncertainties online, and then, the controlled system can be compensated into the nominal model. Then, the command filtered backstepping technique is adopted to deal with the high-order nonlinear system. The stability of the closed-loop 
system is analyzed based on Lyapunov theorem. Simulation results show the effectiveness of the proposed control strategy.

\section{Problem Formulation}

Considering the multi-input-multi-output high order nonlinear system with uncertainties:

$$
\left\{\begin{aligned}
& \dot{\boldsymbol{x}}_{i}=\boldsymbol{f}_{i}\left(\overrightarrow{\boldsymbol{x}}_{i}\right)+\boldsymbol{g}_{i}\left(\overrightarrow{\boldsymbol{x}}_{i}\right) \boldsymbol{x}_{i+1}+\boldsymbol{d}_{i}(t) \quad i=1, \cdots, n-1 \\
& \dot{\boldsymbol{x}}_{n}=\boldsymbol{f}_{n}\left(\overrightarrow{\boldsymbol{x}}_{n}\right)+\boldsymbol{g}_{n}\left(\overrightarrow{\boldsymbol{x}}_{n}\right) \boldsymbol{u}+\boldsymbol{d}_{n}(t) \\
& \boldsymbol{y}=\boldsymbol{x}_{1}
\end{aligned}\right.
$$

where $\boldsymbol{x}_{i} \in \boldsymbol{R}^{m \times 1}$ is system state vector, $\boldsymbol{u}$ is the control input, $\boldsymbol{y} \in \boldsymbol{R}^{m \times 1}$ is output vector , $\boldsymbol{f}_{i}\left(\overrightarrow{\boldsymbol{x}}_{i}\right), \boldsymbol{g}_{i}\left(\overrightarrow{\boldsymbol{x}}_{i}\right)$ is known function, $\boldsymbol{g}_{i}\left(\overrightarrow{\boldsymbol{x}}_{i}\right)$ satisfies Assumption $1, \boldsymbol{d}_{i}(t)$ is the compound disturbance from external disturbances and internal uncertainties. The compound disturbance is assumed satisfying Assumption 2 。

Assumption 1. The matrix $\boldsymbol{g}_{i}\left(\overrightarrow{\boldsymbol{x}}_{i}\right)$ is nonsingular.

Assumption 2. The first order time derivative of the compound disturbance is bounded as:

$$
\dot{d}_{i}(t)=\delta_{i}(t), \sup _{t \in\left[t_{0}, \infty\right)}\left\|\delta_{i}(t)\right\| \leq \delta_{i}
$$

where $\delta_{i}$ is a positive constant.

\section{DOB Based Filtered Backstepping Controller Design}

\subsection{Control Objective}

For the control problem of the high order nonlinear system with uncertainties, the command filter backstepping controller is adopted to reduce the influence from the external noise, thus the controller can be used in practice. The nonlinear DOB is introduced to estimate the compound disturbance online, and compensated in the controller. Figure 1 shows the structure of the control system.

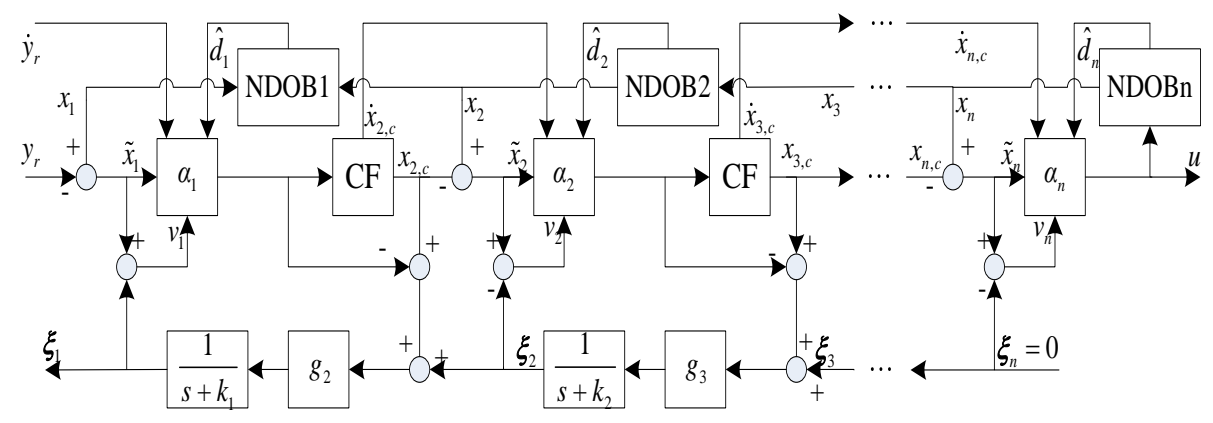

Figure 1. Principle Diagram of the Control System

Control objective: for the MIMO nonlinear system with uncertainties, design the NDOB to estimate the disturbance online, and thus the controller is designed to enable the output $\boldsymbol{y}$ to track the reference signal $\boldsymbol{y}_{r}$ quickly and accurately.

\subsection{NDOB Design}

Theorem 1. For the nonlinear system (1), design the NDOB as follows [9] 
The estimation error of the NDOB satisfies

$$
\left\{\begin{aligned}
\hat{\boldsymbol{d}}_{i}= & \lambda_{i} \boldsymbol{x}_{i}+z_{i} \\
\dot{z}_{i}= & -\lambda_{i}\left(\hat{\boldsymbol{d}}_{i}+\boldsymbol{f}_{i}\left(\overrightarrow{\boldsymbol{x}}_{i}\right)+\boldsymbol{g}_{i}\left(\overrightarrow{\boldsymbol{x}}_{i}\right) \boldsymbol{x}_{i+1}\right) \\
& i=1, \cdots, n-1 \\
\hat{\boldsymbol{d}}_{n}= & \lambda_{n} \boldsymbol{x}_{n}+\boldsymbol{z}_{n} \\
\dot{\boldsymbol{z}}_{n}= & -\lambda_{n}\left(\hat{\boldsymbol{d}}_{n}+\boldsymbol{f}_{n}\left(\overrightarrow{\boldsymbol{x}}_{n}\right)+\boldsymbol{g}_{n}\left(\overrightarrow{\boldsymbol{x}}_{n}\right) \boldsymbol{u}\right)
\end{aligned}\right.
$$

where $0<\mu_{i}<\lambda_{i}$ 。

$$
\left\|\tilde{\boldsymbol{d}}_{i}\right\| \leq \frac{\delta_{i}}{2 \sqrt{\left(\lambda_{i}-\mu_{i}\right) \mu_{i}}}
$$

Proof. Define the estimation error of NDOB as

$$
\tilde{\boldsymbol{d}}_{i}=\hat{\boldsymbol{d}}_{i}-\boldsymbol{d}_{i}
$$

Considering the equation (3), the dynamical equation of the NDOB estimation error is as follows

$$
\begin{aligned}
\dot{\tilde{\boldsymbol{d}}}_{i}= & \dot{\hat{\boldsymbol{d}}}_{i}-\dot{\boldsymbol{d}}_{i}=\lambda_{i} \dot{\boldsymbol{x}}_{i}+\dot{\boldsymbol{z}}_{i}-\dot{\boldsymbol{d}}_{i} \\
= & \lambda_{i}\left[\boldsymbol{f}_{i}\left(\overrightarrow{\boldsymbol{x}}_{i}\right)+\boldsymbol{g}_{i}\left(\overrightarrow{\boldsymbol{x}}_{i}\right) \boldsymbol{x}_{i+1}+\boldsymbol{d}_{i}(t)\right]- \\
& \lambda_{i}\left[\hat{\boldsymbol{d}}_{i}+\boldsymbol{f}_{i}\left(\overrightarrow{\boldsymbol{x}}_{i}\right)+\boldsymbol{g}_{i}\left(\overrightarrow{\boldsymbol{x}}_{i}\right) \boldsymbol{x}_{i+1}\right]-\dot{\boldsymbol{d}}_{i} \\
= & -\lambda_{i}\left[\hat{\boldsymbol{d}}_{i}-\boldsymbol{d}_{i}(t)\right]-\dot{\boldsymbol{d}}_{i}=-\lambda_{i} \tilde{\boldsymbol{d}}_{i}-\dot{\boldsymbol{d}}_{i}
\end{aligned}
$$

Define a Lyapunov function as $V_{\tilde{d}_{i}}=\frac{1}{2} \tilde{\boldsymbol{d}}_{i}^{\mathrm{T}} \tilde{\boldsymbol{d}}_{i}$, according to Assumption 2, we find that $\left\|\dot{\boldsymbol{d}}_{i}\right\| \leq \delta_{i}$, then from Eq (6) we get

$$
\begin{aligned}
\dot{V}_{\tilde{\boldsymbol{d}}_{i}} & =\tilde{\boldsymbol{d}}_{i}^{\mathrm{T}}\left(-\lambda_{i} \tilde{\boldsymbol{d}}_{i}-\dot{\boldsymbol{d}}_{i}\right) \leq-\lambda_{i} \tilde{\boldsymbol{d}}_{i}^{\mathrm{T}} \tilde{\boldsymbol{d}}_{i}+\delta_{i}\left\|\tilde{\boldsymbol{d}}_{i}\right\| \\
& =-2 \lambda_{i} V_{\tilde{\boldsymbol{d}}_{i}}+\delta_{i}\left\|\tilde{\boldsymbol{d}}_{i}\right\| \leq-2\left(\lambda_{i}-\mu_{i}\right) V_{\tilde{\boldsymbol{d}}_{i}}+\frac{\delta_{i}^{2}}{4 \mu_{i}}
\end{aligned}
$$

The estimation error of NDOB satisfies

$$
\left\|\tilde{\boldsymbol{d}}_{i}\right\| \leq \frac{\delta_{i}}{2 \sqrt{\left(\lambda_{i}-\mu_{i}\right) \mu_{i}}}
$$

From Eq. (8), the estimation error of NDOB is uniformly ultimately bounded. With the increasing of the gain of $\mathrm{NDOB}$, the $\mathrm{NDOB}$ converge faster with smaller convergent domain.

\subsection{Filtered Backstepping Based Disturbance Rejection Control}

In the traditional backstepping controller, it is needed to calculate the time-derivative of the virtual control signal, which may cause the calculation explode. Meanwhile, the derivative calculation will make the system sensitive to the noise, which makes the controller hard to be used in practice. The command filtered backstepping defines the following second-order filter: [10]

$$
\left\{\begin{array}{l}
\dot{z}_{i, 1}=\omega_{i} z_{i, 2} \\
\dot{z}_{i, 2}=-2 \varsigma_{i} \omega_{i} z_{i, 2}-\omega_{i}^{2}\left(z_{i, 2}-\alpha_{i}\right)
\end{array}\right.
$$

where $\omega_{i}$ and $\varsigma_{i}$ are the cut-off frequency and damping coefficient, $\alpha_{i}$ is the virtual controller, $z_{i, 1}$ and $z_{i, 2}$ are the estimation of $\alpha_{i}$ and its time-derivative.

At this time, the time-derivative of the virtual controller is acquired by the integration, and the influence caused by noised is reduced. Then, by defining a compensating tracking 
error system, the tracking error of the second-order filter is compensated ${ }^{[10]}$. The compensating tracking error system is defined as follows:

$$
\left\{\begin{array}{l}
\boldsymbol{v}_{i}=\tilde{\boldsymbol{x}}_{i}-\boldsymbol{\xi}_{i} \\
\dot{\boldsymbol{\xi}}_{i}=-k_{i} \boldsymbol{\xi}_{i}+\boldsymbol{g}_{i}\left(\overrightarrow{\boldsymbol{x}}_{i}\right)\left(\boldsymbol{x}_{i+1, c}-\alpha_{i}\right)+\boldsymbol{g}_{i}\left(\overrightarrow{\boldsymbol{x}}_{i}\right) \boldsymbol{\xi}_{i+1}
\end{array}\right.
$$

where $\xi_{i}$ is the tracking error of the command filter, $v_{i}$ is the compensating tracking error, $\boldsymbol{x}_{i+1, c}$ is the tracking error of the virtual controller.

From Eq. (10), $\boldsymbol{v}_{i}$ is the diffeomorphism of the tracking error $\tilde{\boldsymbol{x}}_{i}$, stabilize $\tilde{\boldsymbol{x}}_{i}$ equals to stabilize the virtual tracking error $\boldsymbol{v}_{i}$. Since the tracking error of command filter $\boldsymbol{\xi}_{i}$ is introduced, the stability analysis of $\boldsymbol{v}_{i}$ makes it possible to stabilize $\tilde{\boldsymbol{x}}_{i}$ as well as the command filtered tracking error $\xi_{i}$.

Design the command filtered backstepping controller according to the NDOB estimation result. Introduce the following notations: $\boldsymbol{f}_{i} \square \boldsymbol{f}_{i}\left(\overrightarrow{\boldsymbol{x}}_{i}\right) 、 \boldsymbol{g}_{i} \square \boldsymbol{g}_{i}\left(\overrightarrow{\boldsymbol{x}}_{i}\right)$ 、 $\alpha_{i} \square \alpha_{i}\left(\overrightarrow{\boldsymbol{x}}_{i}, \dot{\boldsymbol{x}}_{i, c}, \hat{\boldsymbol{d}}_{i}\right)$.

Step 1

Consider the first subsystem of the closed-loop system, define the tracking error as

$$
\tilde{\boldsymbol{x}}_{1}=\boldsymbol{x}_{1}-\boldsymbol{y}_{r}
$$

The compound disturbance is estimated by the NDOB

$$
\left\{\begin{array}{l}
\hat{d}_{1}=\lambda_{1} x_{1}+z_{1} \\
\dot{z}_{1}=-\lambda_{1}\left(\hat{d}_{1}+f_{1}+g_{1} x_{2}\right)
\end{array}\right.
$$

The virtual controller is designed as

$$
\alpha_{1}\left(\overrightarrow{\boldsymbol{x}}_{1}, \dot{\boldsymbol{y}}_{r}, \hat{\boldsymbol{d}}_{1}\right)=\boldsymbol{g}_{1}^{-1}\left(-k_{1} \tilde{\boldsymbol{x}}_{1}+\dot{\boldsymbol{y}}_{r}-\boldsymbol{f}_{1}-\hat{\boldsymbol{d}}_{1}-\frac{1}{2 \gamma^{2}} \boldsymbol{v}_{1}\right)
$$

To avoid the derivative calculation, introducing the following filter

$$
\left\{\begin{array}{l}
\dot{z}_{1,1}=\omega_{1} z_{1,2} \\
\dot{z}_{1,2}=-2 \varsigma_{1} \omega_{1} z_{1,2}-\omega_{1}^{2}\left(z_{1,1}-\alpha_{1}\right)
\end{array}\right.
$$

where the initial value of the filter is chosen as $z_{1,1}\left(t_{0}\right)=\alpha_{1}\left(\overrightarrow{\boldsymbol{x}}_{1}, \dot{\boldsymbol{x}}_{1, c}, \hat{\boldsymbol{d}}_{1}\right), z_{1,2}\left(t_{0}\right)=0, t_{0}$ is the initial time.

Define the following compensating tracking error system

$$
\left\{\begin{array}{l}
\boldsymbol{v}_{1}=\tilde{\boldsymbol{x}}_{1}-\boldsymbol{\xi}_{1} \\
\dot{\boldsymbol{\xi}}_{1}=-k_{1} \boldsymbol{\xi}_{1}+g_{1}\left(\boldsymbol{x}_{2, c}-\alpha_{1}\right)+\boldsymbol{g}_{1} \boldsymbol{\xi}_{2}
\end{array}\right.
$$

The initial value is chosen as $\xi_{1}\left(t_{0}\right)=0$, then we get

$$
\begin{aligned}
\dot{\boldsymbol{v}}_{1}=\dot{\boldsymbol{x}}_{1}-\dot{\boldsymbol{\xi}}_{1}=\dot{\boldsymbol{x}}_{1}-\dot{\boldsymbol{y}}_{r}+k_{1} \xi_{1}-\boldsymbol{g}_{1}\left(\boldsymbol{x}_{2, c}-\alpha_{1}\right)-\boldsymbol{g}_{1} \boldsymbol{\xi}_{2} \\
=\boldsymbol{f}_{1}+\boldsymbol{g}_{1} \boldsymbol{x}_{2}+\boldsymbol{d}_{1}-\dot{\boldsymbol{y}}_{r}+k_{1} \xi_{1}-\boldsymbol{g}_{1}\left(\boldsymbol{x}_{2, c}-\alpha_{1}\right)-\boldsymbol{g}_{1} \xi_{2} \\
=\boldsymbol{f}_{1}+\boldsymbol{g}_{1} \alpha_{1}+\boldsymbol{d}_{1}+\boldsymbol{g}_{1}\left(\boldsymbol{x}_{2, c}-\alpha_{1}\right)+\boldsymbol{g}_{1}\left(\boldsymbol{x}_{2}-\boldsymbol{x}_{2, c}\right)-\dot{\boldsymbol{y}}_{r}+ \\
k_{1} \boldsymbol{\xi}_{1}-\boldsymbol{g}_{1}\left(\boldsymbol{x}_{2, c}-\alpha_{1}\right)-\boldsymbol{g}_{1} \xi_{2} \\
=-k_{1}\left(\tilde{\boldsymbol{x}}_{1}-\boldsymbol{\xi}_{1}\right)-\tilde{\boldsymbol{d}}_{1}-\frac{1}{2 \gamma^{2}} \boldsymbol{v}_{1}+\boldsymbol{g}_{1}\left(\tilde{\boldsymbol{x}}_{2}-\boldsymbol{\xi}_{2}\right)
\end{aligned}
$$

where the definition of $\tilde{\boldsymbol{x}}_{2}$ will be given in the next step. With the virtual controller, and the definition of $v_{1}$ and $v_{2}$. It is easy to find that the dynamic equation of the compensation tracking error system is as follows

Step $i$

$$
\dot{v}_{1}=-k_{1} v_{1}-\frac{1}{2 \gamma^{2}} \boldsymbol{v}_{1}+\boldsymbol{g}_{1} \boldsymbol{v}_{2}-\tilde{\boldsymbol{d}}_{1}
$$


Consider the i'th (where $i=1, \cdots, n-1$ ) subsystem of the closed-loop system in Eq. (1), define the tracking error as

$$
\tilde{\boldsymbol{x}}_{i}=\boldsymbol{x}_{i}-\boldsymbol{x}_{i, c}
$$

The compound disturbance is estimated by the NDOB

$$
\left\{\begin{array}{l}
\hat{\boldsymbol{d}}_{i}=\lambda_{i} \boldsymbol{x}_{i}+z_{i} \\
\dot{z}_{i}=-\lambda_{i}\left(\hat{\boldsymbol{d}}_{i}+\boldsymbol{f}_{i}+\boldsymbol{g}_{i} \boldsymbol{x}_{i+1}\right)
\end{array}\right.
$$

According to the command filtered backstepping, the virtual controller is designed as

$$
\alpha_{i}\left(\overrightarrow{\boldsymbol{x}}_{i}, \hat{\boldsymbol{d}}_{i}\right)=\boldsymbol{g}_{i}^{-1}\left(-k_{i} \tilde{\boldsymbol{x}}_{i}+\dot{\boldsymbol{x}}_{i, c}-\boldsymbol{f}_{i}-\boldsymbol{g}_{i-1}^{\mathrm{T}} \boldsymbol{v}_{i-1}-\hat{\boldsymbol{d}}_{i}-\frac{1}{2 \gamma^{2}} \boldsymbol{v}_{i}\right)
$$

where $\boldsymbol{x}_{i, c}$ and $\dot{\boldsymbol{x}}_{i, c}$ are the state $z_{i-1,1}$ and $z_{i-1,2}$ of the filter in the previous step.

To avoid the derivative calculation, introducing the following filter

$$
\left\{\begin{array}{l}
\dot{z}_{i, 1}=\omega_{i} z_{i, 2} \\
\dot{z}_{i, 2}=-2 \varsigma_{i} \omega_{i} z_{i, 2}-\omega_{i}^{2}\left(z_{i, 1}-\alpha_{1}\right)
\end{array}\right.
$$

where the initial value of the filter is chosen as $z_{i, 1}\left(t_{0}\right)=\alpha_{i}\left(\overrightarrow{\boldsymbol{x}}_{i}, \dot{\boldsymbol{x}}_{i, c}, \hat{\boldsymbol{d}}_{i}\right), z_{i, 2}\left(t_{0}\right)=0$.

Define the following compensating tracking error system

$$
\left\{\begin{array}{l}
\boldsymbol{v}_{i}=\tilde{\boldsymbol{x}}_{i}-\boldsymbol{\xi}_{i} \\
\dot{\boldsymbol{\xi}}_{i}=-k_{i} \boldsymbol{\xi}_{i}+g_{i}\left(\overrightarrow{\boldsymbol{x}}_{i}\right)\left(\boldsymbol{x}_{i+1, c}-\alpha_{i}\right)+g_{i}\left(\overrightarrow{\boldsymbol{x}}_{i}\right) \boldsymbol{\xi}_{i+1}
\end{array}\right.
$$

The initial value is chosen as $\xi_{i}\left(t_{0}\right)=0$, then we get

$$
\begin{aligned}
& \dot{\boldsymbol{v}}_{i}=\dot{\tilde{\boldsymbol{x}}}_{i}-\dot{\boldsymbol{\xi}}_{i}=\dot{\boldsymbol{x}}_{i}-\dot{\boldsymbol{x}}_{i, c}+k_{i} \boldsymbol{\xi}_{i}-\boldsymbol{g}_{i}\left(\boldsymbol{x}_{i+1, c}-\alpha_{i}\right)-\boldsymbol{g}_{i} \boldsymbol{\xi}_{i+1} \\
&=\boldsymbol{f}_{i}+\boldsymbol{g}_{i} \boldsymbol{x}_{i+1}+\boldsymbol{d}_{i}-\dot{\boldsymbol{x}}_{i, c}+k_{i} \boldsymbol{\xi}_{i}-\boldsymbol{g}_{i}\left(\boldsymbol{x}_{i+1, c}-\alpha_{i}\right)-\boldsymbol{g}_{i} \xi_{i+1} \\
&=\boldsymbol{f}_{i}+\boldsymbol{g}_{i} \alpha_{i}+\boldsymbol{d}_{i}+\boldsymbol{g}_{i}\left(\boldsymbol{x}_{i+1, c}-\alpha_{i}\right)+\boldsymbol{g}_{i}\left(\boldsymbol{x}_{i+1}-\boldsymbol{x}_{i+1, c}\right)-\dot{\boldsymbol{y}}_{r}+ \\
& k_{i} \boldsymbol{\xi}_{i}-\boldsymbol{g}_{i}\left(\boldsymbol{x}_{i+1, c}-\alpha_{i}\right)-\boldsymbol{g}_{i} \boldsymbol{\xi}_{i+1} \\
&=-k_{i}\left(\tilde{\boldsymbol{x}}_{i}-\boldsymbol{\xi}_{i}\right)-\tilde{\boldsymbol{d}}_{i}-\frac{1}{2 \gamma^{2}} \boldsymbol{v}_{i}+\boldsymbol{g}_{i}\left(\tilde{\boldsymbol{x}}_{i+1}-\boldsymbol{\xi}_{i+1}\right)-\boldsymbol{g}_{i-1}^{\mathrm{T}} \boldsymbol{v}_{i-1}
\end{aligned}
$$

where the definition of $\tilde{\boldsymbol{x}}_{i+1}$ will be given in the next step. With the virtual controller, and the definition of $\boldsymbol{v}_{i}$ and $\boldsymbol{v}_{i+1}$. It is easy to find that the dynamic equation of the compensation tracking error system is as follows

\section{Step $n$}

$$
\dot{\boldsymbol{v}}_{i}=-k_{i} \boldsymbol{v}_{i}-\frac{1}{2 \gamma^{2}} \boldsymbol{v}_{i}+\boldsymbol{g}_{i} \boldsymbol{v}_{i+1}-\tilde{\boldsymbol{d}}_{i}-\boldsymbol{g}_{i-1}^{\mathrm{T}} \boldsymbol{v}_{i-1}
$$

Consider the n'th subsystem of the closed-loop system, define the tracking error as

$$
\tilde{\boldsymbol{x}}_{n}=\boldsymbol{x}_{n}-\boldsymbol{x}_{n, c}
$$

The compound disturbance is estimated by the NDOB

$$
\left\{\begin{array}{l}
\hat{\boldsymbol{d}}_{n}=\lambda_{n} \boldsymbol{x}_{n}+\boldsymbol{z}_{n} \\
\dot{\boldsymbol{z}}_{n}=-\lambda_{n}\left(\hat{\boldsymbol{d}}_{n}+\boldsymbol{f}_{n}+\boldsymbol{g}_{n} \boldsymbol{u}\right)
\end{array}\right.
$$

The controller is defined as

$$
\boldsymbol{u}=\boldsymbol{g}_{n}^{-1}\left(-k_{n} \tilde{\boldsymbol{x}}_{n}+\dot{\boldsymbol{x}}_{n, c}-\boldsymbol{f}_{n}-\hat{\boldsymbol{d}}_{n}-\boldsymbol{g}_{n-1}^{\mathrm{T}} \boldsymbol{v}_{n-1}-\frac{1}{2 \gamma^{2}} \boldsymbol{v}_{n}\right)
$$

where $x_{n, c}$ and $\dot{\boldsymbol{x}}_{n, c}$ are the state $z_{n-1,1}$ and $z_{n-1,2}$ of the filter in the previous step, $\boldsymbol{v}_{n}=\tilde{\boldsymbol{x}}_{n}$, then we get 


$$
\begin{aligned}
\dot{\boldsymbol{v}}_{n} & =\dot{\tilde{\boldsymbol{x}}}_{n}-\dot{\boldsymbol{\xi}}_{n}=\dot{\boldsymbol{x}}_{i}-\dot{\boldsymbol{x}}_{n, c}=\boldsymbol{f}_{n}+\boldsymbol{g}_{n} \boldsymbol{u}+\boldsymbol{d}_{n}-\dot{\boldsymbol{x}}_{n, c} \\
& =-k_{i} \tilde{\boldsymbol{x}}_{n}-\tilde{\boldsymbol{d}}_{n}-\frac{1}{2 \gamma^{2}} \boldsymbol{v}_{n}-\boldsymbol{g}_{i-1}^{\mathrm{T}} \boldsymbol{v}_{i-1}
\end{aligned}
$$

With the controller $u$ and the definition of $\boldsymbol{v}_{n}$, it is easy to find that the dynamic equation of the compensation tracking error system is as follows

$$
\dot{\boldsymbol{v}}_{n}=-k_{n} \boldsymbol{v}_{n}-\frac{1}{2 \gamma^{2}} \boldsymbol{v}_{n}-\tilde{\boldsymbol{d}}_{n}-\boldsymbol{g}_{n-1}^{\mathrm{T}} \boldsymbol{v}_{n-1}
$$

\section{Stability Analysis}

Theorem 2. With the controller (28) and NDOB (13)、(20) and (27), the nonlinear system can be rewritten as the following expression

$$
\left\{\begin{array}{c}
\dot{v}_{1}=-k_{1} \boldsymbol{v}_{1}-\frac{1}{2 \gamma^{2}} \boldsymbol{v}_{1}+\boldsymbol{g}_{1} \boldsymbol{v}_{2}-\tilde{\boldsymbol{d}}_{1} \\
\dot{\boldsymbol{v}}_{i}=-k_{i} \boldsymbol{v}_{i}-\frac{1}{2 \gamma^{2}} \boldsymbol{v}_{i}+\boldsymbol{g}_{i} \boldsymbol{v}_{i+1}-\tilde{\boldsymbol{d}}_{i}-\boldsymbol{g}_{i-1}^{\mathrm{T}} \boldsymbol{v}_{i-1} \\
\quad i=2, \cdots, n-1 \\
\dot{\boldsymbol{v}}_{n}=-k_{n} \boldsymbol{v}_{n}-\frac{1}{2 \gamma^{2}} \boldsymbol{v}_{n}-\tilde{\boldsymbol{d}}_{n}-\boldsymbol{g}_{n-1}^{\mathrm{T}} \boldsymbol{v}_{n-1} \\
\dot{\tilde{\boldsymbol{d}}}_{i}=-\lambda_{i} \tilde{\boldsymbol{d}}_{i}-\dot{\boldsymbol{d}}_{i} \\
\quad i=1, \cdots, n
\end{array}\right.
$$

Consider the closed-loop error system (31), define the following variables

$$
\boldsymbol{z}_{R}=\left[\begin{array}{lll}
p_{1} \boldsymbol{v}_{1}^{\mathrm{T}} & \cdots & p_{n} \boldsymbol{v}_{n}^{\mathrm{T}}
\end{array}\right]^{\mathrm{T}}
$$

where $p_{i}=\sqrt{2\left(k_{i}-\varepsilon_{i}\right)}, \quad k_{i}>\varepsilon_{i}>0$.

Then the $L_{2}$ gain of system in (31) less than $\gamma$.

Proof. From the definition of $z_{R}$

$$
\left\|z_{R}\right\|^{2}=\sum_{i=1}^{n} p_{i}^{2}\left\|\boldsymbol{v}_{i}\right\|^{2}
$$

Define the following Lyapunov function as

$$
V=\frac{1}{2} \sum_{i=1}^{n}\left(\boldsymbol{v}_{i}^{\mathrm{T}} \boldsymbol{v}_{i}+\tilde{\boldsymbol{d}}_{i}^{\mathrm{T}} \tilde{\boldsymbol{d}}_{i}\right)
$$

The time-derivative of this Lyapunov function is as follows

$$
\begin{aligned}
\dot{V} & =-\sum_{i=1}^{n}\left[\left(k_{i}+\frac{1}{2 \gamma^{2}}\right) \boldsymbol{v}_{i}^{\mathrm{T}} \boldsymbol{v}_{i}+\boldsymbol{v}_{i}^{\mathrm{T}} \tilde{\boldsymbol{d}}_{i}+\lambda_{i} \tilde{\boldsymbol{d}}_{i}^{\mathrm{T}} \tilde{\boldsymbol{d}}_{i}+\tilde{\boldsymbol{d}}_{i}^{\mathrm{T}} \dot{\boldsymbol{d}}_{i}\right] \\
& \leq-\sum_{i=1}^{n}\left[\left(k_{i}+\frac{1}{2 \gamma^{2}}\right)\left\|\boldsymbol{v}_{i}\right\|^{2}-\left\|\boldsymbol{v}_{i}\right\|\left\|\tilde{\boldsymbol{d}}_{i}\right\|+\lambda_{i}\left\|\tilde{\boldsymbol{d}}_{i}\right\|^{2}-\delta_{i}\left\|\tilde{\boldsymbol{d}}_{i}\right\|\right]
\end{aligned}
$$

\section{Simulation Result}

Consider the following third order nonlinear system

$$
\left\{\begin{array}{l}
\dot{x}_{1}=x_{2}+d_{1} \\
\dot{x}_{2}=x_{3}+d_{2} \\
\dot{x}_{3}=x_{1} x_{2} x_{3}+u+d_{3}
\end{array}\right.
$$

where the compound disturbance is given as follows 


$$
\left\{\begin{array}{l}
d_{1}=x_{1}^{2} / 20+5 \sin (5 \pi t+\pi / 2) \\
d_{2}=10 \cos (2 \pi t+\pi / 2)+5 t \\
d_{3}=x_{1} x_{2} / 5+25 \sin (0.5 \pi t+\pi / 2)
\end{array}\right.
$$

Assume the initial state is $\left[\begin{array}{lll}0 & 0 & 0\end{array}\right]$, the desired trajectory is given as $y_{r}=10 \sin (2 \pi t+\pi / 2)$, choose the control parameters as $k_{1}=5 、 k_{2}=1 、 k_{3}=1$, and the parameter of the filter is chosen as $\omega_{1}=100 \mathrm{rad} / \mathrm{s} 、 \omega_{2}=50 \mathrm{rad} / \mathrm{s} 、 \varsigma_{1}=1.0 、 \varsigma_{2}=0.5$.

According to the principle of the NDOB, choose the gain of the NDOB as $\lambda_{1}=30$ 、 $\lambda_{2}=10 、 \lambda_{3}=50$. Simulation results are shown in Figure 2, and the estimation effect of the NDOB is shown in Figure 3.

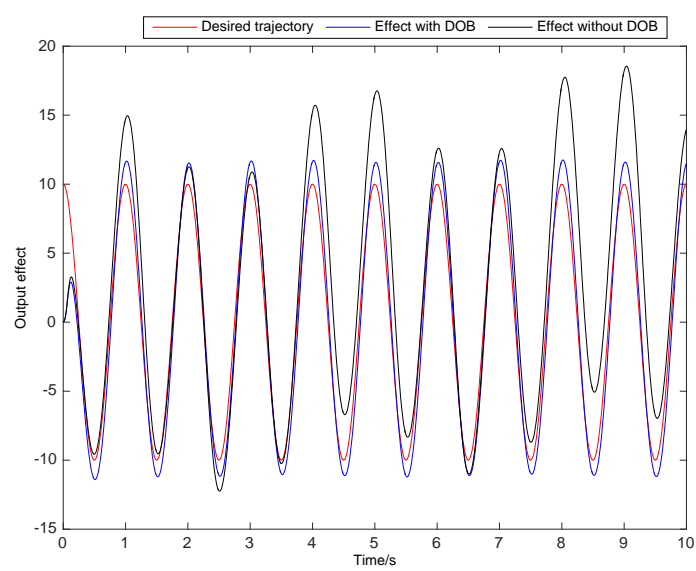

Figure 2. Comparison Control Effect

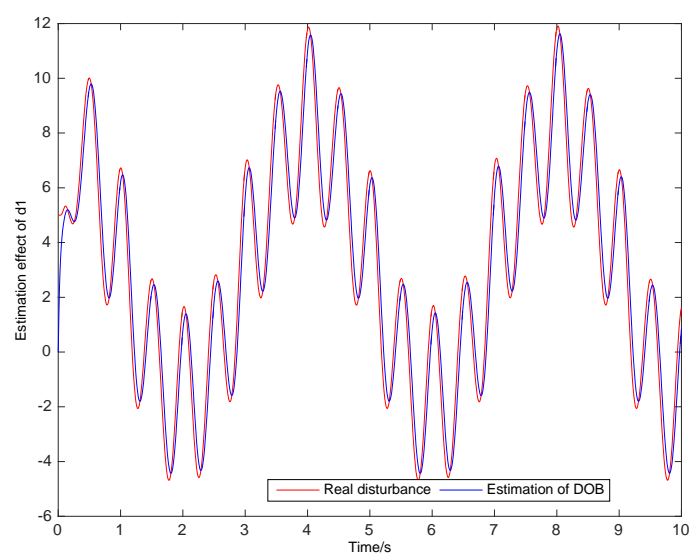

(a) Estimation Effect of $d_{1}$ 


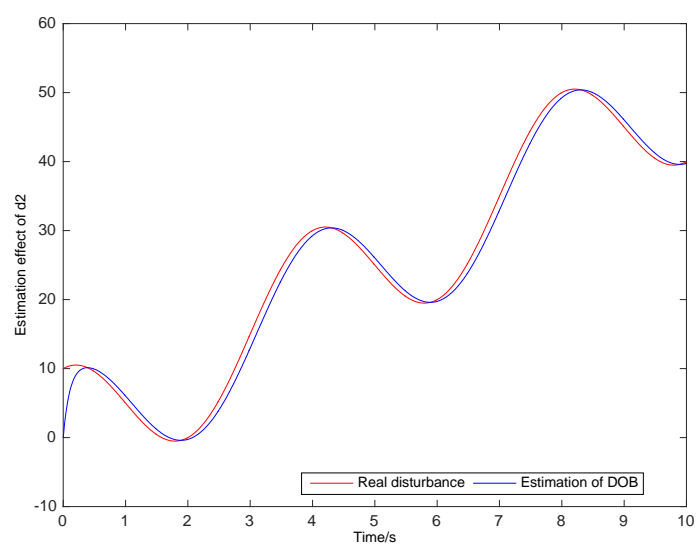

(b) Estimation Effect of $d_{2}$

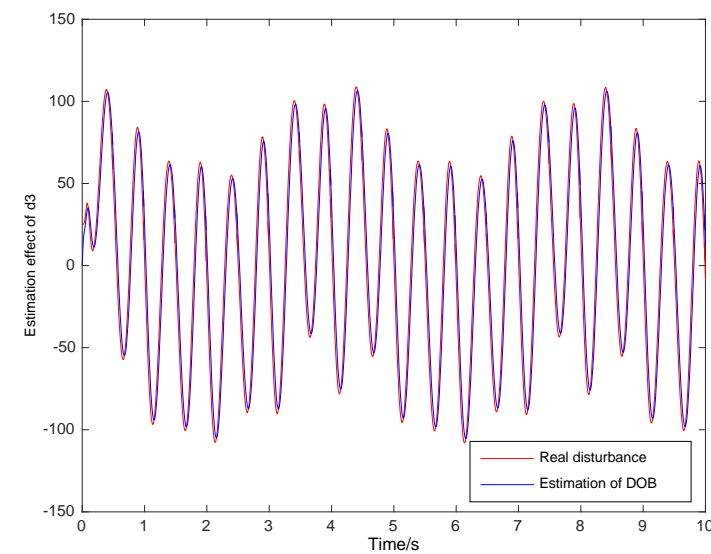

(c) Estimation Effect of $d_{3}$

Figure 3. Estimation Effect of NDOB

It is shown in Figure 2 that, without the NDOB, the control accuracy is largely influenced by the system uncertainties. With the increasing of the disturbances, the tracking error will also increase. For the proposed control methodology, the uncertainties can be estimated by the NDOB, and then compensated in the controller, the control accuracy is much higher than the controller without NDOB. Figure 3 shows that the designed NDOB can estimate the compound disturbance caused by the unmodeled dynamics and external disturbances successfully, the selected observer parameters can enable the estimation error converge quickly. With the NDOB, the nonlinear system with uncertainties can be compensated as the nominal system, thus the controller can be designed to stabilize the certain nominal system.

From the simulation and analysis, the proposed control method can successfully solve the control problem of the high order MIMO system with uncertainties. The command filtered backstepping methodology can deal with the high order system successfully. The designed NDOB can estimate the compound disturbance quickly and accurately.

\section{Conclusion}

For control problem of the high order MIMO nonlinear system with uncertainties, the NDOB based command filtered backstepping methodology is proposed is this paper. The compound disturbance is estimated by the designed NDOB, and thus, the nonlinear system with uncertainties can be compensated into a certain nominal system. Then, the 
command filtered backstepping controller is designed to stabilize the nominal system. The command filtered technique is used to avoid the derivative calculation of high order system. Simulation results show that the proposed method can successfully deal with the control of the high order MIMO system with uncertainties. The proposed controller can enable the system to track a time-varying signal quickly and accurately.

\section{References}

[1] Z. Li, C.-Y. Su, L. Wang, Z. Chen, and T. Chai, "Nonlinear disturbance observer-based control design for a robotic exoskeleton incorporating fuzzy approximation", IEEE Transactions on Industrial Electronics, vol. 62, no. 9, (2015), pp. 5763-5775.

[2] W. Chen, S. S. Ge, J. Wu and M. Gong, "Globally stable adaptive backstepping neural network control for uncertain strict-feedback systems with tracking accuracy known a priori”, IEEE Transactions on Neural Network \& Learning System, vol. 26, no. 9, (2015).

[3] M. Benbouzid, B. Beltran, Y. Amirat, G. Yao and J. Han, "Second-order sliding mode control for DFIGURE-based wind turbines fault ride-through capability enhancement", ISA Transactions, vol. 53, no. 3 , (2014).

[4] Z. Du, D. Yue and S. Hu, "H-infinity stabilization for singular networked cascade control system with state delay and disturbance", IEEE Transactions on Industrial Informatics, vol. 10, no. 2, (2014).

[5] Z. Lianliu and J. Svoboda, "A new control scheme for nonlinear systems with disturbances", IEEE Transactions on Control Systems Technology, vol. 14, no. 1, (2006).

[6] R. Marino, G. L. Santosuosso, and P. Tomei, "Robust Adaptive Observers for Nonlinear Systems with Bounded Disturbances", IEEE Transactions on Automatic Control, vol. 46, no. 6, (2001).

[7] W. H. Chen, D. J. Balance, P. J. Gawthrop and J. O'Reilly, "A Nonlinear Disturbance Observer for Robotic Manipulators", IEEE Transactions on Industrial Electronics, vol. 47, no. 4, (2000).

[8] W. H. Chen, "Disturbance Observer Based Control for Nonlinear Systems", IEEE Transactions on Mechatronics, vol. 9, no. 4, (2004).

[9] K. S. Kim, K. H. Rew and S. Kim, "Disturbance Observer for Estimating Higher Order Disturbances in Time Series Expansion", IEEE Transactions on Automatic Control, vol. 55, no. 8, (2010).

[10] J. A. Farrell, M. Polycarpou, M. Sharma and W. Dong, "Command Filtered Backstepping", IEEE Transactions on Automatic Control, vol. 54, no. 6, (2009). 
International Journal of Control and Automation Vol.10, No.8 (2017) 